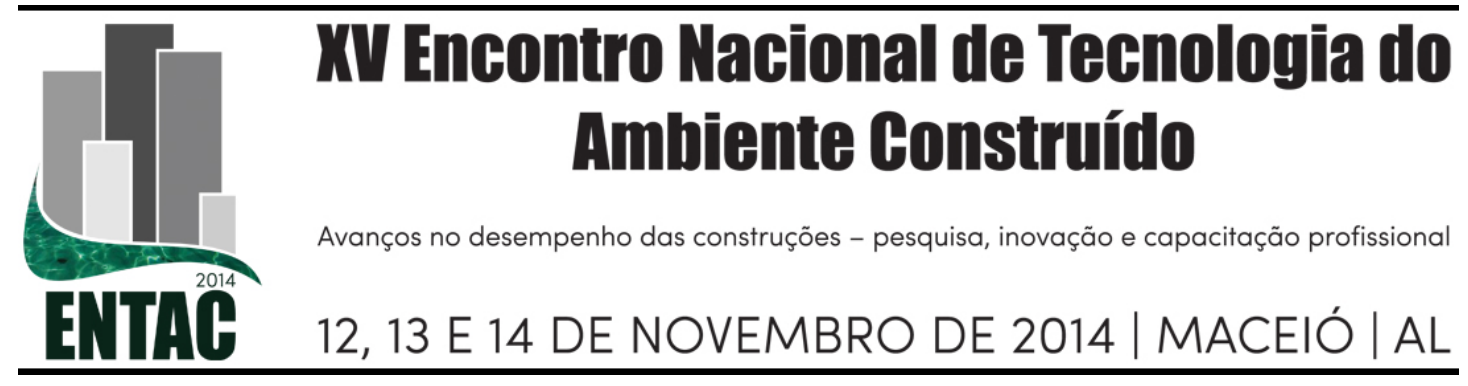

\title{
CLASSIFICAÇÃO ENERGÉTICA DOS MEIOS DE HOSPEDAGEM PELOS REGULAMENTOS TÉCNICOS DA QUALIDADE - RTQS
}

\author{
CRUZ, Juliana (1); INVIDIATA, Andrea (2); FOSSATI, Michele (3); NOVAES, \\ Amadeus de (4); TEXEIRA, Cristiano (5); LAMBERTS, Roberto (6)
}

(1) UFSC, e-mail: julianaycruz@gmail.com (2)UFSC, e-mail: a.invidiata@gmail.com, (3)UFSC, email: michele.fossati@posgrad.ufsc.br (4)UFSC, e-mail: amadeus.mcn@gmail.com (5) UFSC, e-mail: cristiano.andre.teixeira@gmail.com (6) UFSC, e-mail: roberto.lamberts@ufsc.br

\begin{abstract}
RESUMO
O setor hoteleiro tem sido alvo de grandes investimentos nas últimas décadas devido à preparação do Brasil para receber dois eventos esportivos mundiais: a Copa do Mundo de 2014 e os jogos olímpicos de 2016. Segundo um levantamento de 2012, existem no Brasil aproximadamente 9.700 estruturas hoteleiras e só no ano de 2014 tem-se a previsão da abertura de 198 novos hotéis. Da mesma forma, o consumo energético no Brasil vem crescendo rapidamente. Dados do Plano Decenal de Expansão de Energia 2008-2017 afirmam que o consumo energético do setor comercial, incluído o setor hoteleiro, deverá crescer a uma média anual de 6,7\% no período. As maiores participações nos usos finais de energia elétrica do setor hoteleiro se referem à climatização ambiental (41\%) e à iluminação (18\%). Apesar da redução do consumo energético ser de extrema importância, essas iniciativas só terão sucesso se for garantido o conforto interno dos usuários. Mas como avaliar a eficiência energética de um hotel? Atualmente o Regulamento Técnico da Qualidade para o Nível de Eficiência Energética de Edificações Comerciais, de Serviços e Públicas (RTQ-C) enquadra e avalia os meios de hospedagem. Por outro lado, os hotéis apresentam uma diversidade de tipologias, conforme classificadas pelo Sistema Brasileiro de Classificação de Meios de Hospedagem do Ministério do Turismo, algumas mais se assemelhando ao uso residencial do que comercial ou de serviços. Este artigo é resultado de uma pesquisa em desenvolvimento no Centro Brasileiro de Eficiência Energética de Edificações - CB3E e tem como objetivo apresentar propostas de uma nova forma de avaliação da eficiência energética para os diferentes meios de hospedagem segundo os Regulamentos Técnicos da Qualidade para o Nível de Eficiência Energética de Edificações (RTQ-C e RTQ-R). Os pontos positivos e as problemáticas de cada proposta são apresentadas e discutidas.
\end{abstract}

Palavras-chave: Eficiência Energética. Conforto Térmico. Meios de hospedagem. RTQ-R. RTQ-C.

\section{ABSTRACT}

The hotel buildings sector has attracted much investment in the past few years due to the fact that Brazil will host two worldwide sporting events: The FIFA World Cup in 2014 and The 2016 Summer Olympics. According to a 2012 data research, there are 
approximately 9,700 hotels and inns in Brazil, while another 198 of them are under construction, and expected to be finished in 2014. Likewise, the electric energy consumption in Brazil is growing rapidly. The data from the Decennial Plan for Energy Expansion 2008-2017 assert that the electric energy consumption of commercial buildings, including hotels, will increase in average by 6,7\% per year until 2017. The main use of electric energy in the hotel sector is for air conditioning (41\%) and artificial lightning (18\%). Although it is critical to reduce the energy consumption, such initiatives can only succeed if we ensure environmental comfort to the clients. But how do we analyze the energy efficiency of a hotel? Nowadays, we analyze its energy efficiency through the Brazilian Regulation for Energy Efficiency Labeling of Commercial Buildings (RTQ-C). But there are a lot of different categories of hotels, according to a classification by the Ministry of Tourism, and some of their functioning and usage are related more to Residential Buildings than Commercial ones. The research is in progress and the aim of the paper is to present proposals for a new method of analysis of energy efficiency in the hotel sector, considering the Brazilian Regulation for Energy Efficiency Labeling (RTQ-C and RQT-R). The strengths and weaknesses of each proposal are discussed.

Keywords: energy efficiency; thermal comfort; $R T Q-R$; $R T Q-C$; hotels

\section{INTRODUÇÃO}

O consumo de energia elétrica vem aumentando de forma acelerada nos últimos anos, acompanhando o crescimento econômico dos países em desenvolvimento, juntamente com a quantidade de consumidores finais (pessoas e empresas). Estima-se, segundo o International Energy Agency (IEA, 2011), que o consumo energético mundial crescerá $10 \%$ até 2020 , em relação ao consumo atual.

No Brasil, o setor da construção civil também apresentou desenvolvimento que se reflete diretamente na demanda por mais energia elétrica. Mesmo sendo um país em que $42,4 \%$ da energia gerada provêm de fonte renovável (BRASIL, 2013), infelizmente a previsão é que esse número diminua nos próximos anos, aumentando a porcentagem da geração por queima de carvão. Compreendendo essa situação, juntamente com os recentes problemas de sobrecarga nos sistemas de geração e distribuição que acarretaram a falta de energia elétrica no país nos últimos anos, é preciso pensar em alternativas para tornar o consumo mais eficiente.

De acordo com o Balanço Energético Nacional, as edificações são responsáveis por 47,6\% do consumo, sendo divididas em edificações residenciais, comerciais, de serviços e públicas (BRASIL, 2013). Atualmente há dois regulamentos específicos para avaliar a eficiência energética das edificações residenciais e das comerciais, de serviços e públicas: Regulamento Técnico da Qualidade para o Nível de Eficiência Energética de Edificações Residenciais - RTQ-R (BRASIL, 2012) e Regulamento Técnico da Qualidade para o Nível de Eficiência Energética de Edificações Comerciais, de Serviços e Públicas - RTQ-C (BRASIL, 2010). Apesar das edificações hoteleiras serem enquadradas no RTQ-C, seu funcionamento é misto, muitas vezes mais se assemelhando ao uso residencial do que comercial ou de serviços.

O Brasil sediará dois eventos mundiais na área esportiva nos próximos anos (a Copa do Mundo de Futebol em 2014 e as Olimpíadas em 2016). Somente neste ano de 2014, mais 198 hotéis serão inaugurados, num cenário onde já existem $9.700 \mathrm{em}$ todo território nacional (BSH TRAVEL RESEARCH, 2013). Tendo em vista o desenvolvimento do setor e sua importância, acredita-se que deva haver um meio para avaliação da eficiência energética específico para os meios de hospedagem. 


\section{OBJETIVO}

O objetivo deste artigo é apresentar e discutir propostas distintas de avaliação da eficiência energética para os diferentes meios de hospedagem segundo os Regulamentos Técnicos da Qualidade para o Nível de Eficiência Energética de Edificações (RTQ-C e RTQ-R).

\section{CERTIFICAÇÃO ENERGÉTICA EM MEIOS DE HOSPEDAGEM}

O consumo mundial de energia elétrica nos meios de hospedagem está cada vez mais elevado. Nas últimas décadas, a demanda chegou a ser de 97 TWh por ano (STEFAN, 2002). No continente europeu este consumo aproxima-se de $39 \mathrm{TWh}$ por ano, enquanto as emissões de $\mathrm{CO}_{2}$ chegam a 55Mt (BONDANOWICZ e MARTINAC, 2007). Chan (2012) afirma que a causa principal do elevado consumo de energia esteja relacionada com a utilização do sistema de ar-condicionado, ressaltando como as diferenças tipológicas dos meios de hospedagem influenciam este dado. Segundo Ali et al. (2008), o consumo energético no setor hoteleiro varia significativamente de acordo com as características de funcionamento, tipo de serviços oferecidos, operação e caráter de uso, características construtivas e eficiência dos sistemas de condicionamento de ar e de iluminação.

Essa situação também é vista no setor hoteleiro no Brasil, onde o sistema de refrigeração é responsável por $41 \%$ do consumo de energia elétrica, enquanto a iluminação artificial é responsável por $18 \%$ e outros equipamentos, 37\% (SINPHA, 2010).

Nos últimos anos houve um esforço para reverter este cenário por meio de medidas de redução do consumo energético das edificações, o que ajuda a mitigar a pressão sobre a matriz energética e a diminuir a emissão de $\mathrm{CO}_{2}$. Logo, a adoção de uma metodologia de avaliação e classificação ou a certificação energética pode trazer benefícios econômicos e ambientais.

A certificação LEED (Leadership in Energy and Environmental Design) é um sistema internacional de certificação que também avalia edifícios hoteleiros. Criado e administrado pelo GBC (Green Building Council) nos Estados Unidos, o LEED avalia as edificações em quatro níveis: certificado, prata, outro e platina (USGBC, 2009). Na pesquisa de Ahn et al. (2011), os autores apresentam os únicos dois hotéis classificados com a certificação platina (nível máximo). Para meios de hospedagem mais eficientes, que permitiram a obtenção do melhor nível na certificação LEED, as estratégias de eficiência energética foram: sistema de refrigeração de ar altamente eficiente, elevadores com energia alternativa, uso de iluminação artificial com baixo consumo de energia (LED), economia no consumo de água, tecnologias solares (sistemas fotovoltaicos e coletores solares), isolamento térmico da edificação e uso de materiais reciclados. Na sua conclusão afirmam que, através dessas melhorias, os dois estudos de caso obtiveram uma redução de $43 \%$ no consumo de energia, $32 \%$ no consumo de água e, consequentemente, acréscimo de conforto ambiental para o usuário.

Outro programa de certificação energética e sustentável dos meios de hospedagem é a Certificação para a Sustentabilidade Turística (CST, 1996) da Costa Rica. A CST avalia os meios de hospedagem em seis níveis de classificação: do nível 0 (pior desempenho) ao nível 5 (melhor desempenho). Para obter a certificação são analisados: o entorno físico e biológico (interação da empresa turística com o meio e se há programas de proteção do meio ambiente), os serviços oferecidos para o meio de hospedagem (sistemas utilizados para reduzir consumo de energia elétrica e água), a gestão do serviço e a capacidade de incentivo ao hóspede a participar nas políticas de 
sustentabilidade do hotel e o entorno socioeconômico (interação do meio de hospedagem com as comunidades adjacentes).

Há também o programa Chave Verde de Certificação Energética no Setor Hoteleiro, The Green Key (ABAE, 2003). Esse programa nasceu na Dinamarca em 1994, é desenvolvido em 16 países e reconhecido em mais de 30 países. A avaliação dos meios de hospedagem está dividida em 12 áreas temáticas: gestão ambiental, envolvimento dos colaboradores, informação aos clientes, uso da água e energia, higiene e limpeza, tratamento de resíduos, alimentação e bebidas, ambiente interno e externo, atividades ao ar livre e gestão da empresa.

No Brasil, a Norma NBR 15401 determina requisitos para o sistema de gestão de meios de hospedagem que adotam práticas de sustentabilidade. Um hotel para ser sustentável precisa ter uma série de caraterísticas: ter cuidados ambientais, usar fontes de energia renováveis, possuir relacionamento sociocultural com a comunidade onde está inserido, estratégias de economia de água, entre outros.

Além disso, no Brasil existe a avaliação da eficiência energética nos meios de hospedagem realizada pelo Programa Brasileiro de Etiquetagem em Edificações - PBE Edifica, por meio do RTQ-C.

\section{CERTIFICAÇÃO ENERGÉTICA NO BRASIL}

Após a crise de abastecimento de energia em 2001, o Brasil adotou uma nova postura em relação ao consumo de energia. No mesmo ano foi promulgada a Lei $n^{0} 10.295$ (BRASIL, 2001) que dispõe sobre a Política Nacional de Conservação e Uso Racional de Energia, regulamentada pelo Decreto $n^{\circ} 4,059$, de 19 de dezembro de 2001. A Lei da Eficiência Energética, como é conhecida, entre outros resultados, promoveu a regulamentação da eficiência energética de edificações, a partir da publicação dos regulamentos RTQ-C, em vigor desde fevereiro de 2009 e RTQ-R, em vigor desde novembro de 2010.

Os RTQs especificam os requisitos técnicos e o método de classificação do nível de eficiência energética de edificações para obtenção da Etiqueta Nacional de Conservação de Energia (ENCE). A ENCE classifica a eficiência energética das edificações em cinco níveis: de "A" (mais eficiente) a "E" (menos eficiente).

Os regulamentos têm como objetivo determinar os métodos de avaliação e classificação e criar condições para a etiquetagem do nível de eficiência energética de edificações. A etiquetagem de edificações no Brasil ainda é de caráter voluntário, contudo a obrigatoriedade da etiquetagem em nível "A" das edificações públicas federais foi iniciada com a publicação da Instrução Normativa MPOG/SLTI no 02 (BRASIL, 2014).

\subsection{RTQ-C}

O RTQ-C se aplica a edificações com área útil igual ou superior a $500 \mathrm{~m}^{2}$, e/ou com tensão de abastecimento superior ou igual a $2,3 \mathrm{kV}$. São avaliados a envoltória da edificação, o sistema de iluminação e o sistema de condicionamento de ar, cuja classificação geral da edificação é calculada atribuindo-se pesos aos sistemas de $30 \%$, $30 \%$ e $40 \%$, respectivamente. A envoltória é avaliada como um todo, já os sistemas de iluminação e condicionamento artificiais podem ser avaliados parcialmente. Bonificações podem ser somadas à pontuação final, acrescendo até um ponto na nota final da edificação. As bonificações referem-se a sistemas e equipamentos que racionalizem o uso da água; sistemas ou fontes renováveis de energia (aquecimento solar de água, energia eólica e/ou painéis fotovoltaicos); sistemas de cogeração e 
inovações técnicas ou de sistemas que comprovadamente aumentem a eficiência energética da edificação.

Atualmente, as edificações hoteleiras são avaliadas pelo RTQ-C, independente de sua tipologia. Entretanto, alguns meios de hospedagem apresentam um funcionamento misto ou por vezes mais semelhante a uma edificação residencial.

\subsection{RTQ-R}

A classificação da eficiência energética de uma edificação residencial (uni ou multifamiliar) é determinada pela avaliação de dois sistemas: a envoltória e o sistema de aquecimento de água. Além destes, pode ser somado até um ponto na classificação final resultante de bonificações, que são estratégias que aumentam a eficiência da habitação como iluminação e ventilação natural, uso racional de água e uso de equipamentos eficientes. Ao contrário do RTQ-C, o regulamento residencial avalia a envoltória de cada unidade habitacional autônoma (UH) em três diferentes condições: desempenho da envoltória para verão, desempenho da envoltória para inverno e desempenho da envoltória quando condicionada artificialmente.

A avaliação do sistema de aquecimento de água apresenta um peso diferente na ponderação da nota final de acordo com a região geográfica na qual a edificação se localiza, variando de $35 \%$ para as regiões que requerem mais aquecimento e 10 ou $5 \%$ para regiões mais quentes, onde o aquecimento de água não é muito utilizado. $\mathrm{O}$ restante é atribuído à envoltória.

Além da avaliação das unidades habitacionais, o RTQ-R permite a avaliação das áreas de uso comum de edificações multifamiliares e condomínios residenciais. $O$ desempenho energético das áreas de uso comum é determinado pela avaliação das áreas de uso frequente e de uso eventual. São consideradas como áreas de uso frequente: circulações, halls, garagens, escadas, antecâmaras, elevadores, corredores, estacionamento. As áreas de uso eventual são: salões de festa, piscina, brinquedoteca, banheiros coletivos e demais espaços coletivos destinados ao lazer e descanso dos moradores (BRASIL, 2012).

\section{SISTEMA BRASILEIRO DE CLASSIFICAÇÃO DO SETOR HOTELEIRO}

O Sistema Brasileiro de Classificação (SBCLASS, 2008), desenvolvido pelo Ministério do Turismo, é de adesão e adoção voluntárias pelo setor hoteleiro. O SBClass estabelece uma série de requisitos como: infraestrutura, serviços e sustentabilidade. O objetivo é categorizar os meios de hospedagem e definir qual sua tipologia.

Os requisitos são divididos em mandatórios (de cumprimento obrigatório) e eletivos (de livre escolha). O meio de hospedagem, para ser classificado na categoria pretendida, deve ser avaliado por um representante do Inmetro e demonstrar o atendimento a $100 \%$ dos requisitos mandatórios e no mínimo 30\% dos requisitos eletivos (para cada conjunto de requisitos). Os tipos de meios de hospedagem do SBClass são:

I - Hotel: estabelecimento com serviço de recepção, alojamento temporário, com ou sem alimentação, ofertados em unidades individuais e de uso exclusivo dos hóspedes, mediante cobrança diária;

II - Resort: hotel com infraestrutura de lazer e entretenimento que disponha de serviços de estética, atividades físicas, recreação e convívio com a natureza no próprio empreendimento; 
III - Hotel Fazenda: localizado em ambiente rural, dotado de exploração agropecuária, que ofereça entretenimento e vivência do campo;

IV - Cama e Café: hospedagem em residência com no máximo três unidades habitacionais para uso turístico, com serviços de café da manhã e limpeza, na qual o possuidor do estabelecimento resida;

V - Hotel histórico: instalado em edificação preservada em sua forma original ou restaurada, ou ainda que tenha sido palco de fatos histórico-culturais de importância reconhecida;

VI - Pousada: empreendimento de característica horizontal, composto de no máximo 30 unidades habitacionais e 90 leitos, com serviços de recepção, alimentação e alojamento temporário, podendo ser em prédio único com até três pavimentos, ou contar com chalés e bangalôs.

VII - Flat/Apart-hotel: constituído por unidades habitacionais que disponham de dormitório, banheiro, sala e cozinha, em edifício com administração e comercialização integradas, que possua serviço de recepção, limpeza e arrumação.

\section{MÉTODO}

Tendo em vista as sete categorias de hotéis e as diferenças em suas características, o método empregado neste trabalho contempla a apresentação de quatro propostas para avaliação do desempenho energético das diferentes tipologias do setor hoteleiro, elencando suas justificativas e relativas problemáticas em suas aplicações.

\section{RESULTADOS}

As propostas foram elaboradas a partir da análise do panorama atual de certificação de eficiência energética de meios de hospedagem. O presente artigo apresenta diferentes interpretações para a avaliação da eficiência energética através do RTQ-R e RTQ-C das tipologias dos meios de hospedagem. Pretende-se aplicar em um trabalho futuro cada uma das propostas em edificações existentes e definir a melhor interpretação para avaliação de cada tipologia de edificação hoteleira.

A Tabela 1 resume as diferentes propostas apresentadas, indicando por qual método (presente no RTQ-R ou no RTQ-C) deve ser avaliado cada sistema individua.

Tabela 1 - Propostas de avaliação dos meios de hospedagem segundo os RTQs.

\begin{tabular}{|c|c|c|c|c|c|c|}
\hline $\mathbf{N}^{\mathbf{0}}$ & Tipologia & Envoltória & $\begin{array}{c}\text { Sistema de } \\
\text { aquecimento } \\
\text { de água }\end{array}$ & $\begin{array}{c}\text { Sistema de } \\
\text { iluminação } \\
\text { artificial }\end{array}$ & $\begin{array}{c}\text { Condicio- } \\
\text { namento de } \\
\text { ar }\end{array}$ & $\begin{array}{c}\text { Áreas de } \\
\text { uso } \\
\text { comum }\end{array}$ \\
\hline $\mathbf{1}$ & $\begin{array}{c}\text { Todas as } \\
\text { tipologias }\end{array}$ & RTQ-R & RTQ-R & RTQ-C & RTQ-C & $\begin{array}{c}\text { RTQ-R e } \\
\text { RTQ-C }\end{array}$ \\
\hline \multirow{2}{*}{} & $\begin{array}{c}\text { GRUPO 1: Hotéis } \\
\text { e outros com Área } \\
>500 \mathrm{~m}^{2}\end{array}$ & RTQ-C & $\begin{array}{c}\text { RTQ-C } \\
\text { (bonificação) }\end{array}$ & RTQ-C & RTQ-C & RTQ-C \\
\cline { 2 - 7 } & $\begin{array}{c}\text { GRUPO 2: hotel } \\
\text { fazenda, cama } \\
\text { café, flat, pousada }\end{array}$ & RTQ-R & RTQ-R & $\begin{array}{c}\text { RTQ-R } \\
\text { (bonificação) }\end{array}$ & RTQ-R & RTQ-R \\
\hline
\end{tabular}




\begin{tabular}{|c|c|c|c|c|c|c|}
\hline \multirow{3}{*}{} & $\begin{array}{c}\text { GRUPO 1: Flat, } \\
\text { Apart-hotéis }\end{array}$ & RTQ-R & RTQ-R & $\begin{array}{c}\text { RTQ-R } \\
\text { (bonificação) }\end{array}$ & RTQ-R & RTQ-R \\
\cline { 2 - 7 } & GRUPO 2: Outros & RTQ-C & RTQ-R & RTQ-C & RTQ-C & $\begin{array}{c}\text { RTQ-R e } \\
\text { RTQ-C }\end{array}$ \\
\hline $\mathbf{4}$ & $\begin{array}{c}\text { Todas as } \\
\text { tipologias }\end{array}$ & RTQ-C & RTQ-R & RTQ-C & RTQ-C & RTQ-R \\
\hline
\end{tabular}

Nota: Em todas as propostas, há como pré-requisito o refrigerador/frigobar com classificação da ENCE do Inmetro com o nível "A".

Uma das problemáticas que de antemão se destaca para aplicação das propostas é o fato dos regulamentos apresentarem indicadores distintos para avaliar o desempenho energético, impossibilitando uma simples ponderação no cálculo do nível de eficiência energética final. A partir da análise dos resultados de cada regulamento, ao serem aplicadas as propostas, será possível a comparação entre elas e a possível determinação de uma forma mais adequada de ponderação entre RTQ-C e RTQ-R.

\subsection{PROPOSTA 01}

Na primeira proposta elaborada, a avaliação seria feita da mesma forma para as sete tipologias do SBClass, ou seja, os sistemas de iluminação e condicionamento de ar avaliados pelos requisitos do RTQ-C e a envoltória da edificação e o sistema de aquecimento de água avaliados pelos requisitos do RTQ-R.

O sistema de condicionamento de ar representa o maior consumo de energia nos meios de hospedagem. Por ser apenas de caráter informativo no RTQ-R, a análise é mais detalhada se realizada segundo o RTQ-C. O sistema de iluminação artificial é o segundo item responsável pelo consumo de energia em meios de hospedagem, portanto, o sistema seria avaliado segundo o RTQ-C devido ao RTQ-R considerar iluminação artificial somente em bonificações. Com o alto consumo de energia nestes dois sistemas, as áreas de uso comum também teriam estes itens avaliados segundo o RTQ-C, e, caso possuir piscinas e saunas, a avaliação seria através do RTQ-R.

A envoltória seria avaliada segundo o RTQ-R, uma vez que se considera a edificação hoteleira como um conjunto de UHs com características similares a uma residência. E, para aquecimento de água, a análise seria feita através do RTQ-R pois possui requisitos mais detalhados em relação ao RTQ-C.

\subsection{PROPOSTA 02}

Considerando a classificação do setor hoteleiro pelo SBClass, é perceptível que há instalações de hospedagem com características de uso similar a edificações comerciais/de serviço, enquanto outras mais semelhantes às edificações residenciais. Tendo isso em vista, a Proposta 02 divide as tipologias em dois grandes grupos. $\mathrm{O}$ Grupo 01 é constituído de hotel e meios de hospedagem com área superior a $500 \mathrm{~m}^{2}$. O Grupo 02 abrange as demais tipologias: hotel fazenda, cama e café, pousada, hotel histórico, resort e flat. Cada grupo conta com uma divisão distinta entre RTQ-R e RTQC para avaliação dos seus sistemas.

\subsubsection{Grupo 01}

Neste grupo seriam avaliados os meios de hospedagem com caraterísticas semelhantes a edificações comerciais e com grandes superfícies, utilizando-se exclusivamente o RTQ$\mathrm{C}$ (avaliação da envoltória, dos sistemas de iluminação e condicionamento de ar, tanto dos quartos quanto das áreas de uso comum). 
As edificações deste grupo possuem infraestrutura com alto consumo de energia do sistema de refrigeração e iluminação artificial, como, por exemplo, restaurantes, salas de musculação, área para convenções e áreas recreativas.

\subsubsection{Grupo 02}

No Grupo 02, por analisar meios de hospedagem com menor área construída, a avaliação seria feita através do RTQ-R: avaliação da envoltória da edificação, sistema de aquecimento de água e bonificações, assim como as áreas de uso comum.

A escolha de aplicar o regulamento residencial é devido aos quartos, a envoltória e as áreas de uso comum dos meios de hospedagem deste grupo possuírem características semelhantes às UHs de residências e de edificações multifamiliares.

\subsection{PROPOSTA 03}

Da mesma maneira que a Proposta 02, a Proposta 03 considera a classificação do SBClass. Dois grupos são propostos: o Grupo 01 abriga a tipologia dos Flats/Aparthotéis, enquanto Grupo 02 as demais: hotel, resort, hotel fazenda, cama e café, hotel histórico e pousada.

\subsubsection{Grupo 01}

O Grupo 01 seria inteiramente avaliado pelo RTQ-R: avaliação da envoltória da edificação, sistema de aquecimento de água e bonificações, assim como as áreas de uso comum.

A separação da tipologia dos Flats/Apart-hotéis decorre por possuir uso e características geométricas arquitetônicas semelhantes à de uma edificação multifamiliar, e também por possuir uma permanência prolongada por parte dos hóspedes no estabelecimento. Portanto, para verificar tanto a eficiência energética da edificação quanto o conforto dos usuários, essa tipologia e as áreas de uso comum seriam avaliadas segundo o RTQ-R.

\subsubsection{Grupo 02}

No Grupo 02, por predominar edificações semelhantes a edificações comerciais/de serviços, sua avalição seria feita da seguinte maneira: envoltória da edificação, sistemas de iluminação e condicionamento de ar pelo RTQ-C, sistema de aquecimento de água, piscinas e saunas (como área de uso comum, caso existam) pelo RTQ-R.

As tipologias presentes no Grupo 02 possuem menor permanência prolongada por parte do usuário se comparadas com o Grupo 01, portanto, possui uso similar ao de edificações comercial/de serviço.

Ao se analisar o comportamento por parte dos usuários em meios de hospedagens, conclui-se uma diferença significativa comparada às residências. Em edificações de permanência temporária há uso indiscriminado dos sistemas de condicionamento e iluminação artificial, com menor preocupação em relação ao consumo de energia (SBCLASS, 2008).

Caso o meio de hospedagem possua piscinas e/ou saunas na sua infraestrutura, estas seriam avaliadas pelo RTQ-R, como áreas de uso comum.

\section{$7.4 \quad$ PROPOSTA 04}

$\mathrm{Na}$ Proposta 04, todas as tipologias de meios de hospedagem seriam avaliadas da mesma forma: envoltória, sistemas de iluminação e condicionamento artificial nos 
quartos e nas áreas de uso comum pelo RTQ-C e sistema de aquecimento de água, piscinas e saunas pelo RTQ-R.

A justificativa da avaliação dos sistemas serem divididos da maneira apresentada é a mesma da Proposta 03. A diferença nessa proposta é a inclusão dos Flats/Apart-hotéis ao Grupo 02, formando então um grupo único, cujas diferenças tipológicas sugeridas pelo SBClass seriam desconsideradas.

\section{CONCLUSÕES}

Os meios de hospedagem possuem particularidades quando comparadas a outras edificações. As necessidades dos ambientes e do uso final das unidades habitacionais e das áreas de uso comum diferem tanto das edificações residenciais quanto das edificações comerciais e de serviços.

A discussão no presente artigo levanta um questionamento referente à forma com que está sendo abordada a avaliação da eficiência energética em edificações do setor hoteleiro. Por possuir características muitas vezes distintas das edificações comerciais e de serviço, dependendo das características do meio de hospedagem, nem sempre a avaliação pelo RTQ-C é mais adequada. Um exemplo claro é a avaliação de pousadas e pequenos meios de hospedagem pela envoltória como um todo, sem levar em consideração a orientação e o desempenho individual do ambiente. Outro exemplo é a falta de avaliação de áreas de uso comum como piscinas e saunas, geralmente presentes nos meios de hospedagem. Portanto, acredita-se que estas edificações devem possuir uma avaliação que reflita seu real desempenho energético, de acordo com seu uso.

Em decorrência dessas características específicas de cada meio de hospedagem, há a necessidade de desenvolver uma interpretação, tanto do RTQ-C como do RTQ-R, ou mesmo um novo regulamento para que os meios de hospedagem possam ser classificados corretamente quanto ao seu desempenho energético.

A presente pesquisa está sendo desenvolvida e o passo sucessivo será aplicar as propostas elaboradas neste estudo em meios de hospedagens existentes, comparando os pontos positivos e negativos de cada uma delas, assim como os entraves para sua aplicação. A análise dos resultados das propostas possibilitará a criação de uma ferramenta adequada para a avaliação da eficiência energética em meios de hospedagem.

\section{REFERÊNCIAS}

ASSOCIAÇÃO BANDEIRA AZUL DA EUROPA - ABAE. The green Key, 2003. Disponível em: $<$ http://www.abae.pt/programa/GreenKey/inicio.php>. Acesso em: 15 jan. 2014.

ASSOCIAÇÃO BRASILEIRA DE NORMAS TÉCNICAS - ABNT. NBR-15401: Meios de Hospedagem - Sistema de gestão da sustentabilidade - Requisitos. Rio de Janeiro, 2014.

AHN, Y.; CHOI, Y.; PEARCE, A. Sustainable Luxury: Case Study of Two LEED Platinum Hotels. ASCE 2012 - Annual Civil Engineering Conference. 2012.

ALI, Y., MUSTAFA, M., AL-MASHAQBAH, S., MASHAL, K., MOHSEN, M. Potential of energy savings in the hotel sector in Jordan. Energy Conversion And Management, v. 49, n., p.3391-3397, 2008.

BOHDANOWICZ, P., MARTINAC, I. Determinants and benchmarking of resource consumption in hotels - case study of Hilton International and Scandic in Europe. Energy and Buildings, v. 39, n. 1, p. 82-95. 2007. 
BRASIL. Lei $\mathbf{n}^{\mathbf{0}} \mathbf{1 0 . 2 9 5}$, de 17 de outubro de 2001. Dispõe sobre a Política Nacional de Conservação e Uso Racional de Energia, e dá outras providências. In: Diário Oficial da União, Brasília, DF, 2001.

Ministério do desenvolvimento, indústria e comércio exterior. Instituto Nacional de Metrologia, Normalização e Qualidade Industrial - INMETRO. Portaria no $\mathbf{3 7 2}$, de 17 de setembro de 2010. Regulamento Técnico da Qualidade para o Nível de Eficiência Energética de Edificações Comerciais, de Serviços e Públicas (RTQ-C). Disponível em: $<$ http://www.pbeedifica.com.br/etiquetagem/comercial/regulamentos $>$. Acesso em: $28 \mathrm{abr}$. 2014.

Ministério do desenvolvimento, indústria e comércio exterior. Instituto Nacional de $\overline{\text { Metrologia, Normalização e Qualidade Industrial - INMETRO. Portaria no }}{ }^{\mathbf{0}}$ 18, de 16 de janeiro de 2012. Regulamento Técnico da Qualidade para o Nível de Eficiência Energética de Edificações Residenciais (RTQ-R). Disponível em: $<\mathrm{http}$ ///www.pbeedifica.com.br/etiquetagem/residencial/regulamentos $>$. Acesso em: 28 abr. 2014.

Ministério do Planejamento, Orçamento e Gestão. Secretaria de Logística e Tecnologia da Informação. Instrução Normativa n⿳0 02, de 04 de junho de 2014. Dispõe sobre regras para a aquisição ou locação de máquinas e aparelhos consumidores de energia pela Administração Pública Federal direta, autárquica e fundacional, e uso da Etiqueta Nacional de Conservação de Energia (ENCE) nos projetos e respectivas edificações públicas federais novas ou que recebam retrofit. Disponível em: <http://pesquisa.in.gov.br/imprensa/jsp/visualiza/index.jsp?data $=05 / 06 / 2014 \&$ jornal $=1 \&$ pagina $=102 \&$ totalArquivos=164 $>$. Acesso em: 06 jun. 2014 .

Presidência da República. Casa Civil. Subchefia de assuntos jurídicos. Decreto $\mathrm{n}^{\circ}$ $\overline{4.059}$ de 19 de dezembro de 2001. Regulamenta a lei $\mathrm{n}^{\circ} 10.295$, de 17 de outubro de 2001, que dispõe sobre a Política Nacional de Conservação e Uso Racional de Energia, e dá outras providências. In: Diário Ofícial da União. Brasília, DF, 2001. Disponível em: http://www.81.dataprev.gov.br/sislex/paginas/23/2001/4059. htm. Acesso em: 26 abril 2014.

Empresa de pesquisa energética - EPE. Balanço Energético Nacional 2013 - Ano base 2012: Relatório Síntese. Rio de Janeiro, 2013.

BSH TRAVEL RESEARCH, 2013. Investimentos no Brasil: Hotéis \& Resorts. Disponível em: $<$ http://www.bshinternational.com/sys/download/Relat-rio-Investimentos-no-Brasil-2013--Final--1-.pdf $>$. Acesso em: 22 abr. 2014.

CHAN, W. Energy benchmarking in support of low carbon hotels: Developments, challenges, and approaches in China. International Journal of Hospitality Management, v. 31, n. 3, p.1130-1142. 2012.

CST - Certificação para a sustentabilidade turística. Disponível em: $<\mathrm{http}: / / \mathrm{w} w w . t u r i s m o-$ sostenible.co.cr>. Acesso em: 12 mar. 2014. 1996.

INTERNATIONAL ENERGY AGENCY - IEA, 2011. Key World Energy Statistics. Disponível em: <http://www.iea.org/stats/index.asp/>. Acesso em: 15 set. 2013.

SISTEMA BRASILEIRO DE CLASSIFICAÇÃO DE MEIO DE HOSPEDAGEM - SBCLASS, 2008. Disponível em: <www.classificacao.turismo.gov.br/MTUR-classificacao/mtur-site/Sobre. action;jsessionid=3C60DCB77C0F65973A19D737F4EC28AA $>$. Acesso em: 30 fev. 2014.

SISTEMA DE INFORMAÇÃO DE POSSES E HÁBITOS DE USO DE APARELHOS ELÉTRICOS - SINPHA, 2010. Informações Técnicas: Relatório da Pesquisa de Posse de Equipamentos e Hábito de Uso Classe Comercial - AT. Relatório da Classe Comercial - AT - Hotéis/Motéis. Disponível em: <http://www.eletrobras.com.> Acesso em: 30 jan. 2014.

STEFAN, G. Global environmental consequences of tourism. Global Environmental Change, v. 12, n. 4, p. 283-302. 2002.

USGBC. LEED BDC rating system. LEED Building Design + Construction. Disponível em: $<$ http://www. usgbc.org $>$. Acesso em: 06 nov. 2013. 